\title{
RADAR REFLECTION CHARACTERISTICS OF THE MOON
}

\author{
T. B. A. SENIOR AND K. M. SIEGEL \\ University of Michigan, Ann Arbor, Michigan, U.S.A.
}

In recent years many sets of experimental data have been obtained on radar reflections from the moon. After analyzing the results, workers tried to determine the scattering properties of the moon at radar wavelengths, and they concluded that rough-scattering laws could be ascribed to the surface. The available evidence no longer appears to support this view.

This paper presents a theory based on the concept of a "quasi-smooth" scattering mechanism. The main contribution to a radar pulse is provided by a signal that is reflected as though it had come from a smooth body; superimposed on the return are the contributions from other specular areas whose orientation directs a signal báck to the earth. These additional areas may take the physical form of mountains or craters (or parts thereof), and by considering the orientations required of them it is clear that the probability of finding a suitable area at a given distance from the center of the moon's surface will decrease with increasing distance. This fact is born out by the experimental data, which also indicate that the number of significant contributions to the return is comparatively small (no more than ten or so). The moon can, therefore, be treated at radar frequencies as a smooth sphere from which a small number of pieces have been removed to leave facets directed toward the earth.

These conclusions about the nature of the scattering process were arrived at after studying the pulse shapes and power returns obtained by several experimenters at wavelengths from $10 \mathrm{~cm}$ to $2.5 \mathrm{~m}$, and some of the evidence is discussed in sections 1 to 3 . One consequence of the theory is that the initial part of a returned pulse consists entirely of a specular signal arising from a relatively small area in the center of the moon, and power measurements can now be used to determine the electromagnetic constants of part of the moon's surface. The appropriate analysis is given in section 4; in section 5 it is shown that the available data would predict a mean permittivity $8.2 \times 10^{-12}$ farads/meter and a mean conductivity $4.3 \times 10^{-4}$ mhos/meter.

The above theory is of considerable importance in designing a system in which the moon is used as a parasitic antenna to provide communication between widely separated points on the earth's surface. This aspect is briefly discussed in section 6 , and reasons for advocating the use of the $3-\mathrm{cm}$ band for this purpose are given. The relevant parameters of such a system are presented. 


\section{QUASI-SMOOTH MOON THEORY}

Optical experiments over many decades have given a fairly comprehensive picture of the major geographical features of the moon's surface. Visual observations have disclosed the existence of innumerable mountains and craters, and the general impression is that the moon's surface is highly irregular. It is therefore natural to think of the moon as a rough scatterer, and since the intensity of illumination across the face is effectively constant, the moon has been characterized by astronomers as a " rough surface" at optical wavelengths. By this it is implied* that the scattering properties of the moon can be explained in terms of a surface that has a statistically uniform roughness whose scale is measured in microns.

Unfortunately, this theory has led many scientists to assume that roughscattering laws are applicable to the moon at all frequencies, radar as well as optical, and some experimentalists have gone so far as to reject the possibility of using the moon as a means of communication between widely separated points on the earth. Such a conclusion is an unjustified extrapolation from the optical data and, indeed, it is our opinion that the moon does not behave as a rough scatterer at radar frequencies. It is felt that a more accurate description of the surface is "quasi-smooth" (where the meaning to be attached to the new terminology will be made clear later), this view having been reached from a study of the pulse shapes and power returns obtained by several experimenters using a variety of polarizations at wavelengths from $10 \mathrm{~cm}$ to $2.5 \mathrm{~m}$.

One of the reasons for abandoning the roughness concept is provided by the experimental results of Blevis [1]. Working at a frequency of $488 \mathrm{Mc} / \mathrm{s}$ with both $\mathrm{cw}$ and 2-second pulses, and using two orthogonally polarized receivers, Blevis compared the signals scattered from the moon. As regards the long period (Faraday) fades, he found that a maximum signal in one receiver was generally accompanied by a minimum in the other and that, in addition, some of the minima are below the noise level. The existence of maxima and minima is no surprise and is quite in accord with both rough and smooth-scattering theories, but the effective nulls strongly suggest that the second type of scattering process is operating.

To see this, let us consider the radar return from a corner reflector whose sides are very large in respect to a wavelength and upon which there are protuberances whose magnitudes are of order $\lambda / 2$, where $\lambda$ is the wavelength. When orthogonally polarized receivers are used, it is found that the minimum signals always contain at least 30 per cent of the total received energy. On the other hand, if a smooth corner reflector is rotated, the energy contained in a signal at minimum lies between zero and one per cent (the small residual signal being due to edge effects). Which of the two receivers has the minimum depends upon the aspect angle and if the transmitted signal is circularly polarized, the near-zero return is in the receiver with the same

* If the words " rough surface" were to be interpreted as "rugged terrain," the description might well be applicable to the moon. 
(opposite) polarization when the aspect demands an odd (even) number of reflections. Thus, the very fact that the minima in the moon's return can be below the noise level shows that the moon has a reflection property in common with a smooth body.

In view of these results, it is evident that the effective nulls are best explained by assuming that only odd or even numbers of bounces predominate in the return. Since there must obviously be a large single-bounce contribution, it follows that the odd reflections are the major source of the signal. In particular, this requires that the contributions from double bounces be negligible, and strongly suggests that the bulk of the received energy results from single-bounce reflections. Such a statement, however, does not rule out the possibility that double bounces take place, but does imply that the corresponding energy is not usually returned to a receiver on the earth's surface.

These conclusions concerning the nature of the scattering process are in no way contrary to what is known about the moon's topography. To insist that the mere presence of mountains and craters demands a rough-scattering mechanism would also require that a three-sided corner reflector, one of whose sides was measured in millions of wavelengths, while the other two sides were only thousands of wavelengths in dimension, be regarded as a rough surface. In this example the smaller sides are analogous to the mountains on the moon and it is clear that the surface would behave as a smooth reflector. Similarly, a statistical roughness at optical frequencies demands only the existence of irregularities whose scale is measured in microns. A fine sandy surface could provide an appropriate type of roughness, but might well appear smooth at radar frequencies [2].

In the case of radar reflections from the moon, it is clear that there will be some return from mountains and craters superimposed on the smoothsphere signal. These additional contributions will be provided by any smooth areas of the moon suitably oriented to send a signal back to the earth; they could be of the same order of magnitude as the return from the main body of the moon. Since these are also specular in character, the word " quasi-smooth" (rather than smooth) has been used to describe the effect of the moon's surface at frequencies in the meter and centimeter bands.

\section{FAR-ZONE CRITERIA APPLIED TO THE MOON}

An approximate criterion for applying far-zone considerations to (coherent) scattering from a finite two-dimensional body can be obtained by choosing an upper bound for the allowable variation in phase between the returns from individual parts of the surface (assumed perpendicular to the direction of propagation). If it is specified that this variation must be appreciably less than $\pi / 4$ and if, in addition, the dimensions of the receiving antenna are much less than those of the scattering object, the condition becomes

$$
r \gg \begin{gathered}
4 a^{2} \\
\lambda
\end{gathered}
$$


where $r$ is the range and $2 a$ is the maximum dimension of the scatterer. From physical considerations it can be shown that this also holds for a threedimensional body of finite extent if the phase variation is measured as though the scattering were taking place at a plane through the body and perpendicular to the direction of propagation. In other words, it is the body's maximum dimension, rather than its curvature, that places the more stringent condition on the application of far-zone theory.

If $a$ is now identified with the radius of the moon and if the largest value of $\lambda$ is taken to be $5 \times 10^{-3}$ miles, the minimum range at which far-zone theory can be used is $0.8 \times 10^{9}$ miles. This is considerably greater than the moon's actual range (about $2.3 \times 10^{5}$ miles); thus ordinary antenna theory demands that any receiver on the earth be treated as being in the near zone of the moon.

A similar condition to equation (1) can be derived from the exact expression for the field scattered by a sphere on which a plane wave is incident. The expression is in the form of an infinite series of terms whose dependence on range is through spherical Hankel functions (and their first derivatives) of order $\nu$. The coefficients are functions of $k a$, where $a$ is the radius of the sphere, and the summation extends over all (positive) integer values of $\nu$. If the series is now truncated at the term for which $\nu=\nu_{0}$, the error which is incurred will be negligible providing $\nu_{0}>k a>1$. Since the series then contains only a finite number of terms, the Hankel functions can be replaced by the first terms of their asymptotic expansions for large $k r$, and this process is valid if

$$
k r>\nu_{0}\left(\nu_{0}+1\right)>(k a)^{2},
$$

giving

$$
r>\frac{2 \pi a^{2}}{\lambda}
$$

a condition that is in accordance with equation (1). It will be observed that this derivation pays attention to the amplitude as well as the phase, though it is the latter, of course, that is more important when $r>a$.

Let us now consider a pulse scattered by a sphere of radius $a$ at a distance $r$ from the radar. If equation (1) is violated, then at least part of the received pulse will be determined by near-zone scattering laws. On the other hand, the initial part of the pulse of time length $t_{1}$ can only correspond to reflection from the small initially irradiated portion of the sphere. The depth of this zone will be proportional to $t_{1}$ and consequently, as $t_{1}$ increases from zero, the radius of the illuminated area increases from zero to a maximum value $a$. If $t_{1}$ is taken sufficiently small, the part of the pulse for time $t<t_{1}$ must represent a far-zone return, even though the remaining parts of the pulse still require a near-zone treatment.

It is relatively easy to calculate an upper bound for the time interval $t_{1}$ during which far-zone theory can be applied to the moon. If equation (1) is replaced by the less severe condition 


$$
r>\frac{4 a^{2}}{\lambda}
$$

the critical radius for the illuminated zone becomes $\frac{1}{2} \sqrt{r \lambda}$. The depth of this zone on the moon is

$$
d=a-\sqrt{a^{2}-\frac{r \lambda}{4}},
$$

and since $r \lambda$ is much less than $4 a^{2}$,

$$
d \simeq \frac{r \lambda}{8 a}
$$

The corresponding value of $t_{1}$ is then

$$
t_{1} \simeq \frac{r \lambda}{4 a c}
$$

where $c$ is the velocity of propagation in a vacuum; and by inserting the appropriate values for $a$ and $r$ it is found that $t_{1}$ is of the order of 1 microsecond when $\lambda=10 \mathrm{~m}$, decreasing to 0.01 microseconds when $\lambda=10 \mathrm{~cm}$.

To appreciate the importance of this result in connection with the moon, it is convenient to consider the type of pulse that would be received from a perfectly smooth sphere of large radius $a$. There are two cases that have to be discussed. If the range $r$ is such as to satisfy equation (1), the optical scattering cross section is

$$
\sigma=|R|^{2} \pi a^{2},
$$

where $R$ is the voltage reflection coefficient. For a dielectric sphere, this is the leading term in an exact asymptotic expansion proceeding in inverse powers of $(k a)^{2 / 3}$, where $k=2 \pi / \lambda$, and when $k a$ is much greater than unity, equation (3) gives the cross section to a high degree of accuracy. The second case is that in which $r$ violates equation (1). We now confine our attention to the far-zone portion of the returned pulse and providing the dimensions of the corresponding area on the sphere are large compared with the wavelength, optics can again be used to give the scattering cross section of equation (3).

So much for the initial portion of the return. If the transmitted pulse length $\tau$ exceeds $t_{1}$, as will almost certainly be true in applications to the moon, let us measure time $t$ from the instant a return is first received. During the interval $0<t<t_{1}$ equation (3) is valid. Thereafter, a near-zone contribution will be superimposed on the far-zone return, but since the former is of a lower order of magnitude, the over-all level of the signal may be expected to remain essentially unchanged. This will continue until time $t=\tau+t_{1}$. For $t>\tau+t_{1}$ no far-zone contribution is present, and the level of the return will be appreciably reduced, ultimately falling to zero. Nevertheless, the remaining near-zone contributions may persist for several milliseconds beyond time $t=\tau+t_{1}$ and this could well produce some of the pulse elongation observed in the case of the moon. 
The above discussion has been based on the assumption of an entirely smooth moon, and it is clear that the magnitude of the reflection coefficient could be deduced from the initial part of a received pulse in spite of the fact that the moon as a whole is in the near zone. This is still true in a limited sense if the moon is quasi-smooth, though the picture then becomes more complicated. Specular areas other than that in the center of the moon's face will now give rise to contributions and these will generally appear as "spikes" in the tail of the return. With very small values of $\tau$ (a few microseconds only), the shape of the returned pulse during the interval $0<t<\tau+t_{1}$ will not differ appreciably from that for a smooth moon, since the far-zone contributions from individual scattering areas are unlikely to overlap. But as $\tau$ is increased, overlapping will begin to occur and ultimately, for large $\tau$, will have a major effect upon the return, particularly as regards its leading portions. This will be discussed in a moment.

It is important to realize that even if the moon has large regions that are rough at the wavelength under consideration, the contributions from such regions will be of lower magnitude than those from any smooth areas, providing only that the dimensions of the smooth areas are large compared with the wavelength. A consequence of the roughness, however, is to decrease the rate at which the level of the received pulse falls off at the conclusion of the far-zone returns.

\section{THE FORM OF THE REFLECTED PULSES}

On the basis of the theory outlined in the preceding sections, it is possible to predict the general form of a pulse on its return from the moon, surface. To begin with, we shall neglect all disturbances produced by either the moon's motion or by the ionosphere, thereby ruling out the majority of the pulse-to-pulse variations.

On the whole, the energy in the pulse arises from single-bounce reflections at the moon's surface. The initial portion of the pulse is produced by reflection from a relatively small specular area in the center of the moon, and certainly the time at which the signal is received is consistent with this interpretation. In rare cases the moon's aspect may be such that a locally rough patch is situated where the central specular area ought to be; the slope of the initial return will then be smaller and a specular contribution from an adjacent (smooth) area of suitable orientation will dominate the pulse. Similarly, if a mountain were in the precise center of the moon, it would be the first part to give a return, and its different radius of curvature (or reflection coefficient) could lead to yet another variation in the "usual" form of the initial pulse shape.

Additional contributions will come from other specular areas whose orientation directs a signal back to the earth. From a study of the moon's surface it does not seem likely that there will be a large number of these regions and, indeed, the data given in [3] and [4] strongly suggest that the number of major scattering areas is of the order of ten or less. Moreover, by considering the required orientation of scattering areas at different positions on 
the moon's surface, it is immediately obvious that the probability of an area occurring very far from the center of the moon will be comparatively small. As a result, the central region of the moon will be almost entirely responsible for the returned signal and the experimental evidence is in accordance with this. It is estimated that the region is less than one-third of the moon's radius in extent, which rules out any possible return from the moon's limbs. The maximum pulse elongation is thereby reduced from 11 milliseconds to no more than 600 microseconds.

The contributions from these specular areas will appear as "spikes" in the returned pulses, and further information about their location can be deduced from the data obtained by Yaplee et al. using 2-microsecond pulses. Each spike was found to have a width between 2 and 4 microseconds, and from visual observation of the echo, Yaplee concluded that the spacing between spikes was anything from 2 to 4 seconds. In view of the close agreement between the width of the spikes and the transmitted pulse length, it is clear that each spike must be produced by a single specular reflection only. This is confirmed by the way in which spikes in the earlier portions of pulses tend to persist, some for as long as a second. If the spikes were due to constructive interference between the returns from many scattering areas, the libration of the moon would be sufficient to change the interference from one pulse to another, leading to spikes whose positions varied randomly from pulse to pulse. In the later portions of the pulses, however, the spikes do not show as much persistence, which can be explained by the more critical orientation of the corresponding scattering areas as a consequence of their greater displacement from the center of the moon.

The picture we now have is one involving several specular areas concentrated relatively near the moon's center. All these regions produce returns of approximately the same magnitude, which show up as spikes in the echo pulse, and for small values of $\tau$ there is a one-to-one correspondence between the spikes and the specular areas. This enables us to determine the number of areas, and Yaplee's data indicate that there are usually only four or five major contributors. Their separation in depth on the moon's surface, as calculated from the time interval between spikes, is anything from $\frac{1}{2}$ to 5 miles and their surface separation is 30 miles or greater.

The effect of these additional returns on the shape of an echo pulse will depend on the length of the transmitted pulse, and it is convenient to consider separately the cases in which $\tau$ is small (a few microseconds) or large (several hundred microseconds). For values of $\tau$ equal to about 5 microseconds or less, no two scattering areas will be directly illuminated at the same time and the individual specular signals will not then overlap. In particular, the initial portion of the return will be produced entirely by the specular area nearest the earth; since this is usually the main body of the moon (rather than a mountain), the corresponding spike (of width approximately equal to $\tau$ ) will often dominate the whole return.

Other specular areas-at slightly larger distances from the earth will provide similar spikes, and the separation between them probably partially obscures 
the intervening nulls. As a result, these later spikes will appear to constitute a tail to the pulse, but this is not the only source of pulse elongation. At all frequencies under consideration the moon is in the near zone of an antenna on the earth; consequently a relatively large part of the moon will contribute to portions of the pulse at times $t>t_{1}$, where $t_{1}$ is as defined in section 2. This effect is completely independent of the additional specular returns. It would be present even if the moon were entirely smooth. Thus for $t_{1}<t<\tau+t_{1}$ the near-and far-zone effects appropriate to a smooth moon will be superimposed, with the latter appreciably larger than the former, but when $t>\tau+t_{1}$ only the near-zone contributions remain, and their power decreases rapidly with increasing $t$. The spikes characteristic of the later specular reflections will be on top of this tail, and they will serve to raise considerably the power that the tail would otherwise contain.

In the past, the main objection to smooth-moon theory has been based on the feeling that this type of scattering process could not account for the pulse elongation. As stated above, a tail will be produced by a combination of near-zone effect and secondary reflections, but since the duration of the tail will be more or less independent of $\tau$, it is not very apparent when pulse lengths of the order of one millisecond are employed. With short pulses, however, the elongation is a major feature of the return and, for example, Trexler [3] found that with a transmitted pulse 12 microseconds long, only 50 per cent of the received energy appeared in the first 50 microseconds of the echo pulse.

$0 \quad 1020304050 \mathrm{~km}$

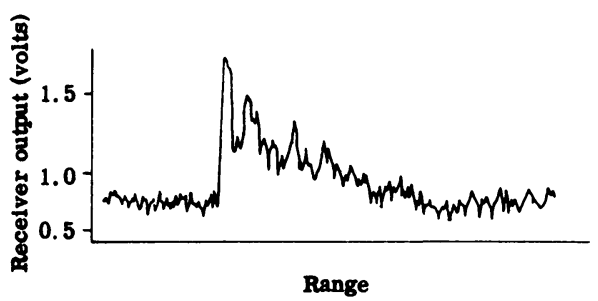

Fig. 1. Amplitude of moon echo.
Still shorter pulses of 5 microseconds' duration have been used by the Royal Radar Establishment, and a diagram showing an integrated return pulse is given in [5]. This is reproduced in Fig. 1 and is in good agreement with the shape predicted by quasismooth-scattering theory. A noticeable feature is the initial sharp rise, which culminates in a narrow peak of width approximately equal to $t_{1}$. Although

this "blip" may or may not be significant, the main lobe has a width closely equal to the pulse length $\tau$, corresponding to the time during which far-zone effects are present. Thereafter, only the near-zone contribution remains and it falls away rapidly with increasing time, disappearing beneath the noise in a time of 150 microseconds. Superimposed on this decreasing return are the expected contributions from specular areas away from the moon's center.

With increasing length of transmitteld pulse, it becomes possible for more than one specular return to be present at the same time, and for sufficiently large $\tau$ there will be portions of the echo pulse to which all the specular areas contribute. The data obtained by Trexler [3] indicate that this occurs if $\tau$ is greater than about 300 microseconds, and it is of interest to consider the type of return to be expected under these circumstances. 
The initial major portion of a returned pulse again corresponds to reflection from the specular area nearest the earth and, to begin with, only this signal is present. When $t$ becomes greater than a few microseconds a further signal will appear, corresponding to the next specular area to be illuminated by the incident pulse, and this will interfere with the first. More and more of these returns will come in as $t$ increases, and the constructive and destructive interference between them will produce a pulse having maxima and minima of a random nature. As a result, the mean level of the echo will increase with time, the actual growth law depending upon the distribution of scattering areas over the surface of the moon and upon the relative magnitude of their individual contributions.

For $t>\tau$, the specular areas that were first illuminated no longer provide returns. The near-zone effect (as for a smooth moon) now assumes importance, though the specular areas that are still illuminated will continue to yield contributions that interfere with one another. As $t$ increases even further, the power level in the return decreases and, ultimately, the pulse will cease to be detectable.

In many respects the complete pulse is similar to that obtained when $\tau$ was small. In particular, the level of the initial part of the significant return is the same in both cases, as is the length of the tail (measured from time $t=\tau)$. On the other hand, the initial return no longer dominates the pulse and the maximum power is achieved at a time $t \simeq \tau$, corresponding to the illumination of all the major scattering areas. A further difference lies in the interpretation of the peaks. The various maxima and minima are now produced by interference and, with the exception of the first and last spikes, cannot be associated with individual scattering areas. Consequently, the positions of the peaks can only be determined by statistical considerations and the motion of the moon will cause them to change from pulse to pulse. Moreover, the number of maxima no longer equals the number of scattering areas.

One important feature of these pulses is the way in which the mean power in the return increases with increasing $t$. It is greatest when $t \simeq \tau$, with the peak return also occurring at about this time. The variation in the maximum received power as the pulse length is changed has been considered by Trexler [6]. He found that for $\tau>300$ microseconds approximately, an increase of $\tau$ was no longer accompanied by an increase in power, and that pulses of this length are therefore sufficient to realize the peak power obtainable with a cw system. The reduction in the maximum which accompanies the use of shorter pulses has been called "modulation loss," and by comparing the results of experiments in which pulses of different lengths were used, Trexler was able to determine values for the modulation loss (relative to a $\mathrm{cw}$ return). Some of these have been communicated by Youmans [7] and show a modulation loss that increases from $1 \mathrm{db}$ for $\tau=200$ microseconds, through $8.5 \mathrm{db}$ at 30 microseconds, and $17 \mathrm{db}$ at 10 microseconds, to $22 \pm 2 \mathrm{db}$ at 2 microseconds. The number of points is not enough to determine accurately the shape of the curve for small values of $\tau$, but it would appear that the loss 
is effectively constant for $\tau<5$ microseconds approximately. With pulses of this duration, the contribution from the first scattering area is isolated from all the rest.

So far in this section, attention has been confined entirely to idealized pulses, which are unaffected by either the moon's motion or the ionosphere. The type of scattering is such that the most significant contributions to the return are produced by relatively small areas on the moon; hence this motion will affect the shape of the received pulses. In particular, the moon's libration will show up as systematic variations in the shape of the tail and, in rare cases, the leading edge of the pulse may also be modified. A study of the pulses should therefore reveal a periodic fluctuation corresponding to the libration, and a fading rate of about $9 \mathrm{c} / \mathrm{s}$, which has been observed in the data, has been ascribed to this cause. The ionosphere also will produce ${ }^{\circ}$ a variety of effects, among which may be listed the variations due to diurnal changes in ionization and to the presence of stray clouds of high ionization density. In addition, the Faraday rotation will lead to periodic fluctuations in the signals received on any one linear polarization. This explanation has been used to account for fades occurring about every two minutes in data obtained at $488 \mathrm{Mc} / \mathrm{s}$ [8].

All these effects tend to mask some of the finer detail in the returns, but in spite of this it is believed that important information about the nature of the moon's surface can be derived from a careful study of the received pulses. In particular, it is possible to determine the reflection coefficient of the specular area nearest the earth and, hence, to calculate the electrical constants of the surface. For this purpose, we recall that the leading edge of the pulse is provided entirely by a specular reflection from the above-mentioned area. If $\tau$ is relatively large, it is difficult to measure this contribution alone, since the pulse is dominated by later peaks formed as a result of interference between many returns of a similar type. For small values of $\tau$, however, the first specular contribution shows up as an initial spike distinct from the rest of the pulse and, since it will usually dominate the other contributions, it is possible to measure the corresponding power with reasonable accuracy. This fact, combined with the greater regularity of the shorter pulses, makes it desirable that only pulses of a few microseconds' duration be employed in any analysis of the moon's surface. Even with such pulses it is still necessary to select those in which the expected initial peak is present and which have not been seriously distorted by influences external to the moon; if this is done, the peak power can be used to calculate the reflection coefficient.

\section{A SIMPLIFIED FORMULA FOR THE REFLECTION COEFPICIENT}

Calculating the electrical constants of the moon's surface from measurements of the moon's return is most easily carried out by a simplified formula for the reflection coefficient, in which the main wavelength dependence is made explicit.

Since the radius of the moon is very much greater than the wavelength 
at all frequencies under discussion, the voltage reflection coefficient $R$ at the specular point is the same as that for an infinite slab of the same material. If a plane electromagnetic wave is incident normally on such a slab,

$$
R=\frac{1-\sqrt{\frac{\mu_{0}}{\mu}\left(\frac{\varepsilon}{\varepsilon_{0}}+i \frac{s}{\omega \varepsilon_{0}}\right)}}{1+\sqrt{\frac{\mu_{0}}{\mu}\left(\frac{\varepsilon}{\varepsilon_{0}}+i \frac{s}{\omega \varepsilon_{0}}\right)}}
$$

(see, for example, [9]), where $\varepsilon, \mu$, and $s$ are the permittivity, permeability, and conductivity, respectively, of the material. The subscript " 0 " denotes the same quantities for free space, and $\omega$ is the frequency. M.k.s. units are employed with a time dependence $\exp (-i \omega t)$.

If the material is primarily dielectric in behavior at the relevant frequencies (as seems a reasonable assumption in the case of the moon), then

$$
s<\omega \varepsilon
$$

and

$$
R \simeq \frac{1-A-i B}{1+A+i B}
$$

where

$$
A=\sqrt{\frac{\mu_{0} \varepsilon}{\mu \varepsilon_{0}}} \quad \text { and } \quad B=\frac{s}{2 \omega \varepsilon_{0}} \sqrt{\frac{\mu_{0} \varepsilon_{0}}{\mu \varepsilon}}
$$

giving

$$
|R|^{2} \simeq \frac{(1-A)^{2}+B^{2}}{(1+A)^{2}+B^{2}}
$$

In the denominator the second term is certainly small compared with the first, hence

$$
|R|^{2} \simeq\left(\frac{1-A}{1+A}\right)^{2}+\left(\frac{B}{1+A}\right)^{2}
$$

Since $\omega=2 \pi c / \lambda$, this equation can be written as

$$
|R|^{2} \simeq C^{2}+\lambda^{2} D^{2}
$$

with

$$
C=\left|\frac{1-A}{1+A}\right|
$$

and

$$
D=\frac{A}{1+A} \frac{s}{4 \pi c \varepsilon}
$$

The expression for the optics scattering cross section is now 


$$
\sigma=\pi a^{2}\left(C^{2}+\lambda^{2} D^{2}\right),
$$

where $a$ is the mean radius of the moon.

In carrying out the analysis leading up to equation (9) several assumptions have been made, which require further explanation. Given the basic assumption that specular reflection takes place (some of the evidence for this has been discussed in section 1), it follows that the radius of curvature in a region surrounding the specular point must be large compared with the wavelength. This in turn justifies the use of a plane slab* to determine an expression for $R$, and since the distance of the moon from the earth is very much larger than the maximum separation of a transmitter and receiver on the surface of the earth, the transmitted field can be treated as a plane wave incident normally on the specular area. Equation (9), however, involves the additional assumption that the radius of curvature is equal to the mean radius of the moon. It is obvious that this may not be true if, for example, the main reflection takes place from a crater, a situation that could arise at particular aspects. In such cases the level of the initial portions of the received pulses will be reduced, ${ }^{\dagger}$ and this suggests that the measured powers to be inserted into equation (9) should be based upon pulses selected for their regular shape and maximum initial rise. If this procedure has not been followed, as may well be the case with the values of $\sigma$ that have already been published, the use of the mean radius in equation (9) must remain a possible source of error.

In the comparison of equation (9) with the experimental data it is natural to assume that constant values of $C$ and $D$ can be used to represent the averages of these quantities over the appropriate frequency range. For the materials of which the moon's surface is composed, it is probable that $\varepsilon, \mu$, and $s$ do not vary rapidly as a function of frequency; but as the wavelength increases, so does the depth to which the incident field penetrates below the surface. The effective reflection takes place at a lower level as $\lambda$ increases, and if the surface layers of the moon are not homogeneous, or if they are stratified, the assumption of a uniform slab in the derivation of $R$ will not be valid. Unfortunately, it is difficult to distinguish a change of material with depth from a frequency variation of $\varepsilon, \mu$, and $s$ for a single substance by using measurements of power only.

Finally, there is the assumption that the moon's surface is primarily dielectric in behavior at the frequencies under consideration. This is certainly true for sufficiently small $\lambda$ but, as $\lambda \rightarrow \infty$, equation (4) shows that $|R|^{2} \rightarrow 1$, whereas equation (6) would predict an infinite value. The precise wavelength at which the approximations break down depends upon the values of $\varepsilon, \mu$, and $s$ and, consequently, the validity of the approximations in the present instance

* An alternative, but entirely equivalent method for obtaining equation (9) would have been to start with the optics expression for the scattering cross section of a dielectric sphere.

$\dagger$ The possibility of focusing occurring has been discarded as being too improbable for consideration here. 
can only be judged after these parameters have been determined. This will be done in the next section.

\section{THE ELECTRICAL CONSTANTS OF THE MOON'S SURFACE}

The value of $\varepsilon, \mu$, and $s$ will now be determined from equation (9) using measured values of the scattering cross section $\sigma$. For this purpose the following data are available:

$$
\begin{array}{lll}
\text { (i) } & \lambda=0.1 \mathrm{~m} & \sigma=0.0003 \pi a^{2} \\
\text { (ii) } \lambda=0.1 \mathrm{~m} & \sigma=0.0004 \pi a^{2} \\
\text { (iii) } \lambda=0.6 \mathrm{~m} & \sigma=0.03 \pi a^{2} \\
\text { (iv) } \lambda=0.7 \mathrm{~m} & \sigma=0.07 \pi a^{2} \\
\text { (v) } \lambda=1.0 \mathrm{~m} & \sigma=(0.05 \sim 0.09) \pi a^{2} \\
\text { (vi) } \lambda=1.5 \mathrm{~m} & \sigma=(0.06 \sim 0.10) \pi a^{2} \\
\text { (vii) } \lambda=2.5 \mathrm{~m} & \sigma=0.1 \pi a^{2}
\end{array}
$$

In interpreting the data, an essential factor is the pulse length. Whereas the measurements at $10 \mathrm{~cm}$ were carried out with pulses of 2 - and 5 -microseconds' duration, all the others are based on the analysis of $\mathrm{cw}$ (or very long pulse) returns, and this accounts for the significantly higher values of the scattering cross section in (iii)-(vii).

The first of the above values of $\sigma$ was obtained by Yaplee et al. [2] at a frequency of $2860 \mathrm{Mc} / \mathrm{s}$ with 2 -microsecond pulses; the sucond is due to the Royal Radar Establishment [10] and is for 5-microsecond pulses. The third and fourth measurements were made by Blevis [1] and Fricker et al. [11] at frequencies of 488 and $413 \mathrm{Mc} / \mathrm{s}$, respectively, using cw systems. The fifth is due to Trexler [3] and has been deduced from his statement that the $\mathrm{cw}$ transmission loss is $258 \mathrm{db}$ when calculated on the basis of isotropic transmitting and receiving antennas. The two values given for $\sigma$ in (v) and (vi) are for a moon at perigee and apogee respectively. The sixth result is the average obtained from many $\mathrm{cw}$ measurements made at or near to a frequency of $200 \mathrm{Mc} / \mathrm{s}$. It has been stated by Trexler [3] as a transmission loss of $254 \mathrm{db}$, but it is of interest to note that Trexler himself found an additional loss of $17 \mathrm{db}$ when using 10 -microsecond pulses at $198 \mathrm{Mc} / \mathrm{s}$. This reduction in power is, of course, the modulation loss. The last value of $\sigma$ was obtained by Evans [12] using 30-millisecond pulses and is a refinement of that originally measured by Browne et al. [13].

In addition to the above, there is a " summary measurement" which indicates the general dependence of the cross section on wavelength. From a review of the data available to him, Trexler [3] states that for frequencies between $20 \mathrm{Mc} / \mathrm{s}$ and $300 \mathrm{Mc} / \mathrm{s}$, the average cw transmission loss increases at a rate of $6 \mathrm{db}$ per octave, passing through the value $258 \mathrm{db}$ at $300 \mathrm{Mc} / \mathrm{s}$. Reference to the radar equation shows that this implies a constant cross section and hence requires that $D$ be zero.

As previously remarked, the values of $\sigma$ given in (iii) through (vii) were determined from either $\mathrm{cw}$, or long-pulse, returns; as such they are measure- 
ments of the power contributed by many individual scattering areas. To calculate a valid reflection coefficient, however, it is necessary to know the power associated with one of these areas alone, and this is only the peak return in the case of very short pulses. It is obviously desirable to restrict the analysis to data obtained with such pulses, but since the number of theşe experiments is relatively small, it becomes necessary to take advantage of all the existing data. To do this, "correction factors" will be applied to the scattering cross sections to reduce them to the values appropriate to microsecond pulses. Using Trexler's figure for the modulation loss associated with a pulse of duration 5 microseconds or less, the modified data is then as follows:

$$
\begin{array}{lll}
\text { (i ) } & \lambda=0.1 \mathrm{~m} & \sigma=0.0003 \pi a^{2} \\
\text { (ii) } & \lambda=0.1 \mathrm{~m} & \sigma=0.0004 \pi a^{2} \\
\text { (iii) } \lambda=0.6 \mathrm{~m} & \sigma=0.0002 \pi a^{2} \\
\text { (iv) } \lambda=0.7 \mathrm{~m} & \sigma=0.0004 \pi a^{2} \\
\text { (v) } \lambda=1.0 \mathrm{~m} & \sigma=0.0004 \pi a^{2} \\
\text { (vi) } \lambda=1.5 \mathrm{~m} & \sigma=0.0005 \pi a^{2} \\
\text { (vii) } \lambda=2.5 \mathrm{~m} & \sigma=0.0006 \pi a^{2}
\end{array}
$$

These results are plotted in Fig. 2. The accuracy of the data is difficult to

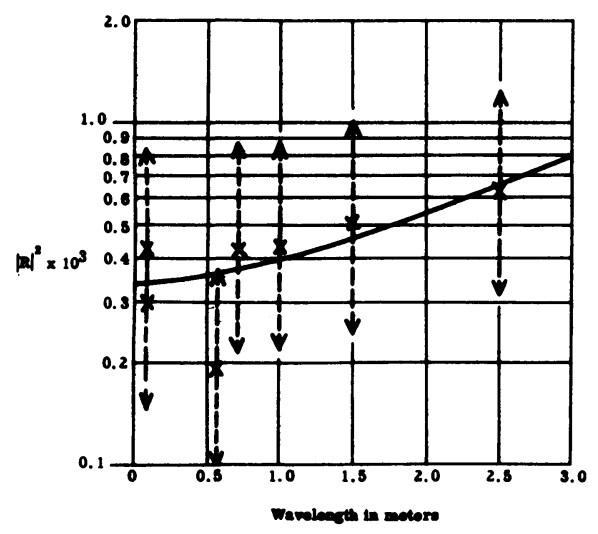

Fig. 2. Comparison between theory and experiment for $|R|^{2}$. assess. Evans has given a probable error of $\pm 3 \mathrm{db}$ for his original measurement, and an uncertainty of $4 \mathrm{db}$ has been attached to the values quoted by Trexler, but in addition to these there are the inaccuracies inherent in trying to deduce the results of a shortpulse experiment from a cw measurement. In the absence of more definite information, we have therefore associated an uncertainty of $\pm 3 \mathrm{db}$ with all the data shown in Fig. 2. It would seem likely that this is an underestimate. Nevertheless, it is not without interest that the slightly lower value of the scattering cross section at $0.6 \mathrm{~m}$ is capable of interpretation in that Blevis obtained his result from a consideration of the mean signal amplitude, rather than the peak return.

If equation (9) is fitted to the modified data using the method of least squares it is found that

$$
C=0.018 \text { and } D=0.0068 \text {, }
$$

and the resulting curve of $|R|^{2}$ against $\lambda$ is given Fig. 2. The corresponding parameters of the moon's surface are 


$$
\begin{aligned}
& \frac{\varepsilon}{\mu}=6.5 \times 10^{-6} \mathrm{mhos}^{2}, \\
& \frac{s}{\mu}=3.4 \times 10^{2} \text { mhos/henry, }
\end{aligned}
$$

and if it is assumed that $\mu$ is the same as for free space, then

$$
\begin{aligned}
& \varepsilon=8.2 \times 10^{-12} \text { farads/meter, } \\
& s=4.3 \times 10^{-4} \text { mhos } / \text { meter } .
\end{aligned}
$$

The phase change on reflection which is implied by these parameters can be determined from equation (4) and the curve is shown in Fig. 3.

Having calculated $\varepsilon$ and $s$, it is possible to test the validity of the approximations leading up to equation (6), and by direct computation of $|R|^{2}$ using equation (4), it can be shown that the approximate formula is accurate to within one per cent over the required range of $\lambda$. Although the curve does not precisely fit the experimental data, the agreement is sufficient to support the theory by which it was obtained. Moreover, the values for $\varepsilon$ and $s$ are in accordance with the hypothesis that the moon's surface is composed of fine sandy material.

\section{COMMUNICATION VIA THE MOON}

The possibility of using the moon as a means of communication between widely separated points on the earth has been largely responsible for the current interest in its scattering properties at microwave frequencies. As early as 1954 it was shown by Trexler [3] that frequency-modulated pulses could be transmitted in this way and, indeed, voice communication at a frequency of $220 \mathrm{Mc} / \mathrm{s}$ was successfully carried out.

A major factor in the design of such systems is the need to obtain received pulses of a regular and consistent shape with the minimum of frequency distortion. If the moon were to behave as a rough scatterer, a more regular return could be produced by using increasingly large wavelengths, the ultimate limit being given by the cut-off frequency of the ionosphere. On the other hand, a smooth-scattering mechanism implies that the leading edge of the pulse will suffer less frequency distortion at the shorter wavelengths, since the contributing area on the moon is then smaller. This is particularly true for very short pulses. With this fact in

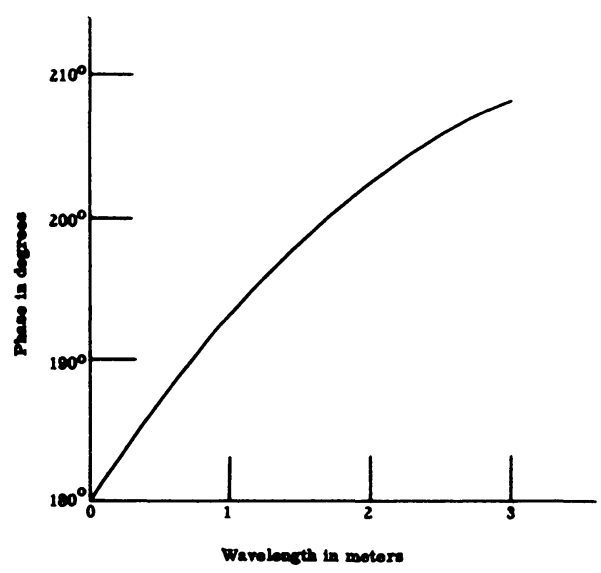

FIG. 3. Phase change on reflection. 
mind, the theory presented in this paper would suggest an FM communication system operating at very high frequencies and employing only the initial portions of the received pulses.

There are several other advantages attached to high frequencies. As the frequency is increased, the ionospheric effects are drastically reduced. Under this heading may be grouped the disturbances due to meteor trails and the Faraday rotation of the plane of polarization. The latter shows itself as a systematic fading of a linearly polarized signal and can, if necessary, be avoided by using circular polarization. The rotation, however, decreases with increasing frequency and is considerably less than one degree at $10,000 \mathrm{Mc} / \mathrm{s}$.

Originally it was felt that the reflection coefficient of the moon would fall off appreciably at high frequencies, and this was one reason why wavelengths of the order of a meter were suggested for communication systems. It has now been shown that the reduction is relatively small (no more than $3 \mathrm{db}$ ), and since the power gain of an antenna of given size is proportional to $\lambda^{-2}$, a significant increase in the returned power can be achieved by decreasing the wavelength.

All these considerations favor the highest possible frequencies. Probably the only limit is provided by oxygen and water-vapor absorption. When coupled with the desire to use standard equipment, a natural choice is 10,000 $\mathrm{Mc} / \mathrm{s}$. On this basis we would advocate a frequency-modulated pulsed communication system with approximately the following characteristics:

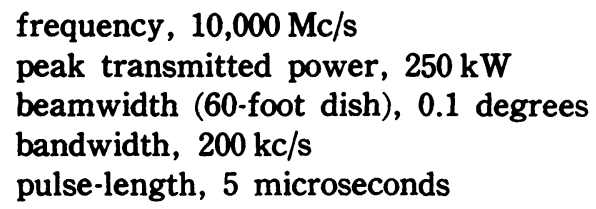

The moon return would be about $10^{-13}$ watts, giving a signal-to-noise ratio of about $10 \mathrm{db}$ with a receiver having a noise figure of 11 . The information rate could be as large as that attained during transmission bursts with meteorscatter systems, but now, of course, uninterrupted transmission is possible for as long as the moon is visible to both the transmitter and the receiver.

\section{CONCLUSIONS}

The theory of a quasi-smooth moon was arrived at from a study of many sets of experimental data, and it is felt that the pulse shapes and power returns can be satisfactorily explained on this basis, as can their polarization dependence. The initial part of a received pulse takes on a particular significance, in that it consists entirely of a specular return. Moreover, only one specular area contributes toward it, and if pulses of very short duration are employed, the contribution shows up as a peak, which can be distinguished from the remainder of the pulse. This enables power measurements to be employed to determine the electromagnetic constants of the relevant portion of the moon's surface; the values so obtained lend credence to this method.

The accuracy of the existing data is not really sufficient to justify a guess 
at the actual materials that make up the specular region of the moon's surface. Consequently, it is desirable that further measurements be carried out to improve the accuracy, paying particular attention to the power contained in the initial part of the pulse. The use of very narrow-beam antennas would further localize the area of the moon's surface that contributes to this portion of the return.

In addition these results can be applied to the design of communication systems that employ scattering from the moon. After considering the factors affecting such a system, it is possible to conclude that appreciable advantages are attached to using the highest possible frequencies, the ultimate limit being provided only by the increasing atmospheric absorption at wavelengths of a few centimeters.

The authors wish to acknowledge the assistance and cooperation of the following: J. C. W. Scott and B. C. Blevis of the Radio Physics Laboratory, Defence Research Board, Canada; J. H. Trexler, B. S. Yaplee, and A. B. Youmans of Naval Research Laboratory; R.L. Leadabrand of Stanford Research Institute; A.S. Gross of the U.S. Army Signal Research and Development Laboratories; J.S. Hey and V. A. Hughes of Royal Radar Establishment, England; J. V. Evans of Manchester University, England; F. T. Haddock, B. A. Harrison, and $\mathrm{H}$. Weil of The University of Michigan.

The work reported in this paper was carried out under contract with the Rome Air Development Center of the Air Research and Development Command, United States Air Force.

\section{REFERENCES}

[1] Blevis, B. C. Personal communications (1957 October 8 and 1958 June 3).

[2] Grant, C. R., and Yaplee, B. S. Proc. I.R.E. 45, 976, 1957.

[3] Trexler, J. H. Proc. I.R.E. 46, 286, 1958.

[4] Yaplee, B. S., Bruton, R. U., Craig, K. J., and Roman, N. G. Proc. I.R.E. 46, 293, 1958.

[5] The Staff of the Royal Radar Establishment. Nature, 180, 1225, 1957.

[6] Trexler, J. H. Personal communication (1958 June 4).

[7] Youmans, A. B. Personal communication (1958 June 6).

[8] Scott, J. C. W. Personal communication (1957 May 24).

[9] Stratton, J. A. Electromagnetic Theory. New York (McGraw-Hill Book Co., Inc.), 1941.

[10] Hughes, V. A. Personal communication (1958 April 28).

[11] Fricker, S. J., Ingalls, R. P., Mason, W. C., and Stone, M. L. Paper presented at Joint URSI-IRE Meeting, Washington, 1958.

[12] Evans, J. V. Proc. Phys. Soc. Lond., B 70, 1105, 1957.

[13] Browne, I. C., Evans, J. V., Hargreaves, J. K., and Murray, W. A. S. Proc. Phys. Soc. Lond., B 69, 901, 1956.

\section{Discussion}

F. G. Smith: I would like to give my reasons for rejecting the approach to this problem taken by Siegel and Senior. I refer first to the analysis made in Paris by Fresnel in the last century, in which he gave the justification 
for using geometric optics in reflection from a plane mirror. Qualitative consideration of Fresnel diffraction theory suggests that the optical approach is entirely justified, even for radio reflections from a perfectly spherical reflecting mirror of the size and distance of the moon.

The echoes in fact show that all parts of the return, including the initial pulse, suffer Rayleigh fading, and may be considered as coming from many small areas between which there is no coherence. The criteria applied by Siegel and Senior for different types of diffraction theory can therefore have no relevance.

Senior and Siegel [submitted at the request of the editor]: Dr. Smith comments on two quite separate points. The first is concerned with scattering by a perfectly conducting smooth sphere, and his point appears to be that simple Fresnel theory is adequate to analyze a returned pulse.

It is certainly true that for the scattering of a plane wave by a sphere, Fresnel theory would predict the leading term of the asymptotic expansion of the exact solution as given in equation (3). The theory, however, cannot be accepted as proving the presence (or otherwise) of a tail in a returned pulse. An example is provided by the axially symmetric scattering by a perfectly conducting paraboloid. The exact (pulse) solution shows that a returned pulse has no tail, whereas Fresnel theory applied to the exact Kirchhoff-Huygens integral would suggest that the successive zones on the paraboloid give contributions to the pulse whose amplitude decays slowly only with increasing time. In this case, Fresnel theory would appear to predict a tail whose existence is denied by exact theory. In short, Fresnel theory is of doubtful validity in any discussion of the tail in a returned pulse, and it is improbable that Fresnel himself would have sought to use it in such circumstances.

The comments in Dr. Smith's second paragraph are based on his hypothesis that the moon behaves as an essentially rough reflector at radar wavelengths. This is not in accordance with the results in, for example, the paper of Yaplee and his colleagues. 